PROCEEDINGS OF THE

AMERICAN MATHEMATICAL SOCIETY

Volume 129, Number 5, Pages 1403-1408

S 0002-9939(00)05811-1

Article electronically published on October 25, 2000

\title{
POROUS SETS THAT ARE HAAR NULL, AND NOWHERE APPROXIMATELY DIFFERENTIABLE FUNCTIONS
}

\author{
JAN KOLÁr̆
}

(Communicated by David Preiss)

\begin{abstract}
We define a new notion of "HP-small" set $A$ which implies that $A$ is both $\sigma$-porous and Haar null in the sense of Christensen. We show that the set of all continuous functions on $[0,1]$ which have finite unilateral approximate derivative at a point $x \in[0,1]$ is HP-small, as well as its projections onto hyperplanes. As a corollary, the same is true for the set of all Besicovitch functions. Also, the set of continuous functions on $[0,1]$ which are Hölder at a point is HP-small.
\end{abstract}

By S. Banach [2] and S. Mazurkiewicz 8], the set of nowhere differentiable functions is residual in $\mathcal{C}([0,1])$. P. M. Gandini and A. Zucco [5] and V. Anisiu [1] have shown that its complement $A$ is even $\sigma$-porous. V. Anisiu also considered functions with finite unilateral approximate derivative at a point. A better porosity constant was obtained by D. L. Renfro 9 .

More recently, B. R. Hunt [6] proved that $A$ is Haar null (in the sense of Christensen). The main aim of this paper is to define a new property stronger than both $\sigma$-porosity and Haar nullness and to show that the set $A$ has this property. We will also extend Hunt's result to functions with finite unilateral approximate derivative at a point.

\section{HP-SMALL SETS}

Let $A$ be a subset of a Banach space $X$ and $c \in[0,1]$. We say that $A$ is $c$-globally very porous if for every $c^{\prime} \in(0, c), x \in X$ and $r>0$ there is a ball $B=B\left(y, c^{\prime} r\right)$, $y \in \overline{B(x, r)}$, such that $B \cap A=\emptyset$. A set $A$ is $\sigma$-c-globally very porous if it is a countable union of $c$-globally very porous sets. The number $c$ will be called the porosity constant.

If $A$ is $(\sigma-) c$-globally very porous, then it is easy to see that $A$ is $(\sigma-)$ globally very porous in the sense of [10] and $(\sigma$ - $) \alpha$-globally very porous in the sense of [9] with $\alpha=2 c /(1+c)$.

A Borel subset $A$ of a separable Banach space $X$ is said to be Haar null [4] if there is a Borel probability measure $\mu$ on $X$ so that $\mu(A+x)=0$ for every $x \in X$. Let us extend this definition: a general $A \subset X$ is called Haar null if it is a subset

Received by the editors August 9, 1999.

1991 Mathematics Subject Classification. Primary 26A27, 28C20, 26A16, 26A24.

Key words and phrases. Typical continuous function, $\sigma$-porous sets, Haar null sets, approximate derivative, Besicovitch functions, nowhere Hölder functions.

The author was supported by the grants GAUK 165/99 and CEZ:J13/98:113200007. 
of a Borel set with the same property. The following characterization is due to E. Matoušková.

Proposition 1 ([7]). Let $X$ be a separable Banach space and $A \subset X$ a Borel set. Then $A$ is Haar null if and only if for every $\delta>0$ and $r>0$ there exists a Borel probability measure $\mu$ with $\operatorname{spt} \mu \subset \overline{B(0, r)}$ such that $\mu(A+x) \leq \delta$ for every $x \in X$.

Definition 2. Let $A$ be a subset of a Banach space $X$ and $c \in(0,1]$. We say that $A$ has property $H P_{(c)}$ if for every $c^{\prime} \in(0, c)$ and $r>0$ there exists $K>0$ and a sequence of balls $\left\{B_{i}\right\}=\left\{B\left(y_{i}, c^{\prime} r\right)\right\}$ with $\left\|y_{i}\right\| \leq r, i \in \mathbf{N}$, such that for every $x \in X$,

$$
\operatorname{card}\left\{i \in \mathbf{N}:\left(x+B_{i}\right) \cap A \neq \emptyset\right\} \leq K .
$$

The set $A$ is said to be HP-small if there is a porosity constant $c \in(0,1]$ such that $A$ is a countable union of sets with property $\mathrm{HP}_{(c)}$.

HP-small sets obviously form a $\sigma$-ideal. When we say that the HP-typical $x \in X$ has certain property we mean that the set of $x \in X$ without this property is HPsmall in $X$.

Proposition 3. Every HP-small subset of a Banach space is $\sigma$-c-globally very porous (where $c \in(0,1]$ is the porosity constant) and hence meager.

Every HP-small subset of a separable Banach space is Haar null.

Proof. Let $c \in(0,1]$ and let $A$ be a set with property $\mathrm{HP}_{(c)}$. Since $\bar{A}$ also has property $\mathrm{HP}_{(c)}, A$ may be assumed to be closed. Let $c^{\prime} \in(0, c), r>0$ and $\delta>0$ be given. Let $\left\{B_{i}\right\}=\left\{B\left(y_{i}, c^{\prime} r\right)\right\}$ be a sequence of balls as in the Definition. Choose $n>K / \delta$ and let $\mu=\frac{1}{n} \sum_{i=1}^{n} \delta_{y_{i}}$. Obviously spt $\mu \subset \overline{B(0, r)}$ and $\mu(x+A) \leq K / n<$ $\delta$ for every $x \in X$. Thus $A$ is a Haar null set (by Proposition 1) and so is every HP-small set since the Haar null sets are closed under countable unions 4 .

Also, at least one of the balls $x+B\left(y_{i}, c^{\prime} r\right)$ is disjoint with $A$ and this is enough to verify that $A$ is $c$-globally very porous.

Theorem 4. Let $A$ be an HP-small subset of a Banach space $X$ and let $B \subset X$ be $a \sigma$-compact set. Then the set $A+B=\{a+b: a \in A, b \in B\}$ is HP-small.

Proof. This is a consequence of the following proposition.

Proposition 5. Let $X$ be a Banach space, $c \in(0,1], A \subset X$ a set with property $H P_{(c)}$ and $B \subset X$ a compact set. Then $A+B$ has property $H P_{(c)}$.

Proof. Let $c^{\prime} \in(0, c)$ and $r>0$ be given. Choose an arbitrary $c_{A}^{\prime} \in\left(c^{\prime}, c\right)$ and let $\varepsilon=r\left(c_{A}^{\prime}-c^{\prime}\right)$. By compactness of $B$, there exist $z_{1}, \ldots, z_{n} \in X$ such that $B \subset \bigcup_{j=1}^{n} B\left(z_{j}, \varepsilon\right)$. Since $A$ has property $\mathrm{HP}_{(c)}$, there is $K_{A}>0$ and $y_{1}, y_{2}, \ldots \in X$, $\left\|y_{i}\right\| \leq r$ such that for every $x_{A} \in X$,

$$
\operatorname{card}\left\{i \in \mathbf{N}:\left(x_{A}+B\left(y_{i}, c_{A}^{\prime} r\right)\right) \cap A \neq \emptyset\right\} \leq K_{A} .
$$

Now if $x \in X$ and $\left(x+B\left(y_{i}, c^{\prime} r\right)\right) \cap(A+B) \neq \emptyset$, then there is $z \in B$ such that $\left(x+B\left(y_{i}, c^{\prime} r\right)\right) \cap(A+z) \neq \emptyset$ and hence there is $j \in\{1, \ldots, n\}$ such that $\left(x-z_{j}+B\left(y_{i}, c^{\prime} r+\varepsilon\right)\right) \cap A \neq \emptyset$. Using the identity $c^{\prime} r+\varepsilon=c_{A}^{\prime} r$ and (10) with $x_{A}=x-z_{j}$ separately for each $j \in\{1, \ldots, n\}$, we have

$$
\operatorname{card}\left\{i \in \mathbf{N}:\left(x+B\left(y_{i}, c^{\prime} r\right)\right) \cap(A+B) \neq \emptyset\right\} \leq K
$$

where $K=n K_{A}$. 
Examples. If $X$ is an infinite dimensional Banach space and $A=\{0\}$ is a singleton in $X$, then $A$ has property $\mathrm{HP}_{\left(\frac{1}{2}\right)}$. If moreover $X=\ell_{2}$ or $X=\mathcal{C}([0,1])$, then $A$ has property $\mathrm{HP}_{(1)}$. By Proposition 5 the same is true for any compact set $A$.

Obviously, if $X$ and $Y$ are Banach spaces, $M$ is an HP-small subset of $X$ and $p \in[1, \infty]$, then $M \times Y$ is an HP-small subset of $X \oplus_{p} Y$. Thus $A=\{0\} \times Y$ and $B=X \times\{0\}$ are HP-small in $X \oplus_{p} Y$ provided $\operatorname{dim} X=\operatorname{dim} Y=\infty$. However, $A+B=X \times Y$ is not HP-small, so we cannot replace " $\sigma$-compact" by "HP-small" in Theorem 4 .

Corollary 6. Under the hypotheses of Theorem 4, there exists $x \in X$ such that $A \cap(x+B)=\emptyset$. Moreover, this is true for the HP-typical $x \in X$.

Proof. $\{x \in X: A \cap(x+B) \neq \emptyset\}=A-B$ is HP-small.

Corollary 7. If $X$ is an infinite dimensional Banach space and $A \subset X$ is $H P$ small and homogeneous (i.e. $\lambda A=A$ for every $\lambda \neq 0$ ), then there exist linearly independent $y_{1}, y_{2}, \ldots \in X$ such that $\operatorname{span}\left\{y_{1}, y_{2}, \ldots\right\} \subset(X \backslash A) \cup\{0\}$.

Proof. By induction, when $y_{1}, y_{2}, \ldots, y_{n-1}$ with $A_{n-1}=\operatorname{span}\left\{y_{1}, y_{2}, \ldots, y_{n-1}\right\} \subset$ $(X \backslash A) \cup\{0\}$ have been constructed, we use Corollary 6 and take an arbitrary $y_{n} \in X \backslash A_{n-1}$ such that $A \cap\left(y_{n}+\operatorname{span}\left\{y_{1}, y_{2}, \ldots, y_{n-1}\right\}\right)=\emptyset$.

Assume that there is a linear combination $y=\sum_{i=1}^{n} a_{i} y_{i} \in A_{n} \cap A$ with $a_{n} \neq 0$. Then $y_{n}+\sum_{i=1}^{n-1} \frac{a_{i}}{a_{n}} y_{i} \in A \cap\left(y_{n}+\operatorname{span}\left\{y_{1}, y_{2}, \ldots, y_{n-1}\right\}\right)=\emptyset$, a contradiction. Thus $A_{n} \subset(X \backslash A) \cup\{0\}$ for every $n \in \mathbf{N}$.

Remark. It is possible to proceed with induction over all ordinals $n \in\left[1, \omega_{1}\right)$ to obtain a linear subspace $E \subset(X \backslash A) \cup\{0\}$ of uncountable dimension. Also, $E$ can be found dense in $X$ provided $X$ has a dense subset of cardinality $\leq \omega_{1}$.

Proposition 8. Let $A$ be a subset of a Banach space $X$. If $P: X \rightarrow X$ is a continuous linear projection with $\operatorname{dim} \operatorname{Ker} P<\infty$, then the following are equivalent:

(i) A is HP-small;

(ii) $P(A)$ is HP-small in $X$;

(iii) $P(A)$ is $H P$-small in $P(X)$.

Proof. Since $A \subset P(A)+\operatorname{Ker} P, P(A) \subset A+\operatorname{Ker} P$ and $\operatorname{Ker} P$ is $\sigma$-compact, (i) and (ii) are equivalent by Theorem 4 The implication (iii) $\Longrightarrow$ (ii) follows directly (with porosity constant $\|P\|$-times smaller) from the definition and from the inclusion $\left(x+B\left(y_{i}, c^{\prime} r\right)\right) \cap P(X) \subset P(x)+B\left(y_{i},\|P\| c^{\prime} r\right)$ for $x \in X$ and $y_{i} \in P(X)$. The inclusion is valid since $\left\|z-\left(x+y_{i}\right)\right\| \leq c^{\prime} r$ and $z \in P(X)$ implies

$$
\left\|z-\left(P(x)+P\left(y_{i}\right)\right)\right\| \leq\|P\| c^{\prime} r .
$$

It remains to prove (ii) $\Longrightarrow$ (iii). We may assume that $A=P(A) \subset P(X)$ and $A$ has property $\mathrm{HP}_{(c)}$ in $X(c \in(0,1])$. Let $c^{\prime} \in(0, c)$ and $r_{0}>0$ be given. Let $r=r_{0} /\|P\|$ and $B=\operatorname{Ker} P \cap B(0,(1+\|P\|) r)$. In the same way as in the proof of Proposition 5 we obtain $y_{1}, y_{2}, \ldots \in X,\left\|y_{i}\right\| \leq r$ and $K$ such that (2) holds true for every $x \in X$. Let $\tilde{y}_{i}=P\left(y_{i}\right)$ for $i \in \mathbf{N}$. Then for $\tilde{x} \in P(X)$,

$$
B\left(\tilde{x}+\tilde{y}_{i}, c^{\prime} r\right) \cap A \neq \emptyset \quad \Longrightarrow \quad B\left(\tilde{x}+y_{i}, c^{\prime} r\right) \cap(A+B) \neq \emptyset
$$

because $y_{i}-\tilde{y}_{i} \in \operatorname{Ker} P$ and $\left\|y_{i}-\tilde{y}_{i}\right\| \leq\left\|y_{i}\right\|+\left\|P\left(y_{i}\right)\right\| \leq(1+\|P\|) r$. Hence for every $\tilde{x} \in P(X)$

$$
\operatorname{card}\left\{i \in \mathbf{N}: B\left(\tilde{x}+\tilde{y}_{i}, \frac{c^{\prime}}{\|P\|} r_{0}\right) \cap A \neq \emptyset\right\} \leq K .
$$


Since $\tilde{y}_{i} \in P(X)$ and $\left\|\tilde{y}_{i}\right\| \leq\|P\| r=r_{0}$, this shows that $A$ has property $\operatorname{HP}_{(c /\|P\|)}$ in $P(X)$.

Examples. By the proposition, no closed hyperplane is HP-small, although it is both Haar null and 1-globally very porous. The only HP-small subset of $\mathbf{R}^{d}$ is the empty set.

If $X=Y \oplus_{t} E$, with $\operatorname{dim} E<\infty$, and $F: Y \rightarrow E$ is an arbitrary function, then the graph of $F$ is not HP-small in $X$.

\section{HP-TYPICAL CONTINUOUS FUNCTIONS}

Recall that if $\lambda$ denotes the Lebesgue measure in $\mathbf{R}$ and $A \subset \mathbf{R}$ is measurable, then the right lower density of $A$ at $x \in \mathbf{R}$ is defined as

$$
\underline{\mathrm{d}}_{+}(A, x)=\liminf _{h \rightarrow 0+} \frac{\lambda(A \cap(x, x+h))}{h} .
$$

If $f$ is a measurable function then the approximate right upper limit of $f$ at $x$ is the number

$$
\limsup _{y \rightarrow x+} \operatorname{ap} f(y)=\inf \left\{a: \underline{\mathrm{d}}_{+}(\{y: f(y) \leq a\}, x)=1\right\} .
$$

The approximate right lower limit is defined similarly. If they are equal, then the approximate right limit is the common value. The approximate left limit and the unilateral approximate derivatives are defined in the standard manner.

We say that $f:[0,1] \rightarrow \mathbf{R}$ is nowhere Hölder if for all $x \in[0,1]$ and $\alpha>0$

$$
\sup _{y \in[0,1]} \frac{|f(y)-f(x)|}{|y-x|^{\alpha}}=\infty \text {. }
$$

Theorem 9. The HP-typical $f \in \mathcal{C}([0,1])$ is nowhere Hölder and has a finite unilateral approximate derivative at no point.

The Theorem immediately follows when the following stronger result is applied with $\phi(t)=1 / \max (-\ln t, 1)$.

Proposition 10. Let $\phi:(0, \infty) \rightarrow(0, \infty)$ be a nondecreasing function such that $\lim _{t \rightarrow 0+} \phi(t)=0$. Then the HP-typical $f \in \mathcal{C}([0,1])$ has

$$
\limsup _{y \rightarrow x+} \frac{|f(y)-f(x)|}{\phi(|y-x|)}=\infty
$$

for each $x \in[0,1)$. (Moreover the porosity constant is 1 .)

Proof. Choose arbitrary $\frac{1}{16}<\alpha<\alpha^{\prime}<\frac{1}{8}$ (so that $\alpha^{\prime}<2 \alpha$ ). Assume that (3) does not hold for an $x \in[0,1)$. Then its left-hand side is smaller than some $K \in \mathbf{N}$ and

$$
\underline{\mathrm{d}}_{+}(\{y:|f(y)-f(x)| \leq K \phi(|y-x|)\}, x)=1 .
$$

Therefore there exists $n \in \mathbf{N}$ such that $x \in[0,1-1 / n]$ and

(4) $\lambda(\{t \in(0, h):|f(x+t)-f(x)|>K \phi(t)\}) \leq \alpha h \quad$ for every $h \in(0,1 / n)$.

Let us denote by $A_{n, K}$ the set of all continuous functions satisfying (4) for an $x \in[0,1-1 / n]$. To prove the Proposition, it is enough to show that for each $K, n \in \mathbf{N}$ the set $A_{n, K}$ has property $\mathrm{HP}_{(1)}$ in $\mathcal{C}([0,1])$.

Fix $n, K \in \mathbf{N}, c^{\prime} \in(0,1), r>0$. Choose an $l=2^{-i_{0}}, i_{0} \in \mathbf{N}$, such that $l<\frac{1}{2 n}$ and $K \phi(2 l)<r\left(1-c^{\prime}\right)$. For $i \in \mathbf{N}$ let $\tilde{f}_{i}=r \operatorname{sgn}\left(\sin 2^{i} \pi x\right)$ and find an $f_{i} \in \mathcal{C}([0,1])$, 
$\left\|f_{i}\right\| \leq r$ so that $\lambda\left(\left\{f_{i} \neq \tilde{f}_{i}\right\}\right) \leq\left(\frac{1}{4}-2 \alpha^{\prime}\right) l$. For each interval $I \subset[0,1]$ of length $l$ and for $i, j>i_{0}, i \neq j$ we have

$$
\begin{aligned}
& \lambda\left(\left\{y_{1} \in I: f_{i}\left(y_{1}\right)=r=-f_{j}\left(y_{1}\right)\right\}\right) \geq 2 \alpha^{\prime} l, \\
& \lambda\left(\left\{y_{2} \in I:-f_{i}\left(y_{2}\right)=r=f_{j}\left(y_{2}\right)\right\}\right) \geq 2 \alpha^{\prime} l .
\end{aligned}
$$

The proof will be finished as soon as we show that for every $f \in \mathcal{C}([0,1])$

$$
\operatorname{card}\left\{i \in \mathbf{N}: B\left(f+f_{i}, c^{\prime} r\right) \cap A_{n, K} \neq \emptyset\right\} \leq i_{0}+\frac{\alpha}{\alpha^{\prime}-\alpha} \frac{1}{l}+1 .
$$

Assume that $f$ is such that this is not true. Let $M$ be the set of all $i>i_{0}$ such that there exists $g_{i} \in B\left(f+f_{i}, c^{\prime} r\right) \cap A_{n, K}$. For $i \in M$ let $x_{i} \in[0,1-1 / n]$ be such that (cf. (41))

$$
\lambda\left(\left\{t \in(0, h):\left|g_{i}\left(x_{i}+t\right)-g_{i}\left(x_{i}\right)\right|>K \phi(t)\right\}\right) \leq \alpha h \quad \forall h \in(0,1 / n) .
$$

Since (6) is assumed to be false, card $M>\frac{\alpha}{\alpha^{\prime}-\alpha} \frac{1}{l}+1$ and there exist $i, j \in M$, $i \neq j$, such that $\left|x_{i}-x_{j}\right| \leq \frac{\alpha^{\prime}-\alpha}{\alpha} l$. Assume $x_{i} \leq x_{j}$ and let $I=\left[x_{j}, x_{j}+l\right]$.

Relations (5) provide us with $y_{1}, y_{2} \in I$ satisfying $f_{i}\left(y_{1}\right)=r=-f_{j}\left(y_{1}\right)$, $-f_{i}\left(y_{2}\right)=r=f_{j}\left(y_{2}\right)$ and

$$
\begin{aligned}
\left|g_{i}\left(y_{m}\right)-g_{i}\left(x_{i}\right)\right| & \leq K \phi\left(y_{m}-x_{i}\right) \leq K \phi(2 l), \\
\left|g_{j}\left(y_{m}\right)-g_{j}\left(x_{j}\right)\right| & \leq K \phi\left(y_{m}-x_{j}\right) \leq K \phi(l),
\end{aligned}
$$

for $m=1,2$. To make this more precise, let us fix $m \in\{1,2\}$. By (7) with $h=\left(x_{j}+l\right)-x_{i}=l+\left|x_{j}-x_{i}\right| \leq l\left(1+\frac{\alpha^{\prime}-\alpha}{\alpha}\right)=\frac{\alpha^{\prime}}{\alpha} l \leq 1 / n$, the set $\left\{y_{m} \in I\right.$ : (8) is not true $\}$ has measure at most $\alpha h \leq \alpha^{\prime} l$. Similarly (with $h=l$ and $i$ replaced by $j),\left\{y_{m} \in I\right.$ : (9) is not true $\}$ has measure at most $\alpha l<\alpha^{\prime} l$. Together these two sets have measure less than $2 \alpha^{\prime} l$ and we can apply the $m$-th row of (5) to obtain $y_{m}$.

By (8),,$\left|g_{i}\left(y_{1}\right)-g_{i}\left(y_{2}\right)\right| \leq 2 K \phi(2 l)$ and by (9), $\left|g_{j}\left(y_{1}\right)-g_{j}\left(y_{2}\right)\right| \leq 2 K \phi(l) \leq$ $2 K \phi(2 l)$. Hence

$$
\left|\left(g_{i}-g_{j}\right)\left(y_{1}\right)-\left(g_{i}-g_{j}\right)\left(y_{2}\right)\right| \leq 4 K \phi(2 l)<4 r\left(1-c^{\prime}\right) .
$$

Since $\left\|g_{i}-\left(f+f_{i}\right)\right\| \leq c^{\prime} r$ and $\left\|g_{j}-\left(f+f_{j}\right)\right\| \leq c^{\prime} r$, we have

$$
4 r=\left|\left(f_{i}-f_{j}\right)\left(y_{1}\right)-\left(f_{i}-f_{j}\right)\left(y_{2}\right)\right|<4 r\left(1-c^{\prime}\right)+4 r c^{\prime}=4 r .
$$

Thus (16) must be true.

We have the following corollary for Besicovitch functions, that is, for functions with no finite or infinite unilateral derivative at any point.

Corollary 11. The HP-typical $f \in \mathcal{C}([0,1])$ has an infinite unilateral derivative on a set having cardinality of the continuum. Thus Besicovitch functions form an $H P$-small subset of $\mathcal{C}([0,1])$.

The proof of the Corollary is exactly the same as Preiss' simple proof of Saks' theorem (see [3, Ch. 15, 3.2.1 and 3.2.2]). (We use Theorem 9 as well as the fact that the set of nondecreasing functions is HP-small in $\mathcal{C}([0,1])$ since they are differentiable almost everywhere.)

\section{ACKNOWLEDGMENT}

The author would like to thank Professor L. Zajíček for his inspiring questions and for suggesting Corollary 11 


\section{REFERENCES}

1. V. Anisiu, Porosity and continuous, nowhere differentiable functions, Ann. Fac. Sci. Toulouse Math. (6) 2 (1993), 5-14. MR 94h:26007

2. S. Banach, Über die Baire'sche Kategorie gewisser Funktionenmengen, Studia Math. 3 (1931), 174-179.

3. A. Bruckner, Differentiation of Real Functions [2nd edition], CRM Monograph Series, Providence, Rhode Island, 1994. MR 94m:26001

4. J. P. R. Christensen, Topology and Borel structure, North-Holland, Amsterdam, 1974. MR 50:1221

5. P. M. Gandini and A. Zucco, Porosity and typical properties of real-valued continuous functions, Abh. Math. Sem. Univ. Hamburg 59 (1989), 15-22. [MR 91c:26007]

6. B. R. Hunt, The prevalence of continuous nowhere differentiable functions, Proc. Amer. Math. Soc. 122 (1994), 711-717. MR 95d:26009

7. E. Matoušková, The Banach-Saks property and Haar null sets, Comment. Math. Univ. Carolinae 39 (1998), 71-80. MR 99g:46013

8. S. Mazurkiewicz, Sur les fonctions non dérivables, Studia Math. 3 (1931), 92-94.

9. D. L. Renfro, Some supertypical nowhere differentiability results for $\mathcal{C}[0,1]$, Doctoral Dissertation, North Carolina State University, 1993.

10. L. Zajíček, Porosity and $\sigma$-porosity, Real Anal. Exchange 13 (1987-88), 314-350. MR 89e:26009

Department of Mathematical Analysis, Charles University, Sokolovská 83, 18675 Praha 8, Czech Republic

E-mail address: kolar@karlin.mff.cuni.cz 